S. Besset and J-J. Sinou, Modal reduction of brake squeal systems using complex interface modes, Mechanical Systems and Signal Processing, 85, 896-911, 2017.

doi:10.1016/j.ymssp.2016.09.006

\title{
Modal reduction of brake squeal systems using complex interface modes
}

\author{
S. Besset ${ }^{\mathrm{a}, \mathrm{b}, \mathrm{d}}$, J.-J. Sinou ${ }^{\mathrm{a}, \mathrm{b}, \mathrm{c}, \mathrm{e}}$ \\ ${ }^{a}$ Laboratoire de Tribologie et Dynamique des Systèmes, UMR CNRS 5513, École Centrale de Lyon, 36 \\ avenue Guy de Collongue 69134 Écully Cedex, France \\ ${ }^{b}$ Centre Lyonnais d"Acoustique, Université de Lyon, 69622 Lyon, France \\ ${ }^{c}$ Institut Universitaire de France, 75005 Paris, France \\ ${ }^{d}$ sebastien.besset@ec-lyon.fr \\ ejean-jacques.sinou@ec-lyon.fr
}

\begin{abstract}
This paper deals with a new efficient reduction method for predicting the stability analysis of a damped nonlinear brake system subjected to friction-induced vibration. The originality of the present work is to propose a generalized double modal synthesis method that combines a classical modal reduction and a condensation at the frictional interface by computing a reduced complex mode basis.

Comparisons with the existing double modal synthesis reduction method are performed. It is demonstrated that the new suggested reduction technique is more efficient. It allows to provide satisfactory results with a small number of interfaces modes.
\end{abstract}

Keywords: friction-induced vibration, double modal synthesis, brake squeal

\section{Nomenclature}

M: Mass matrix of the system

K: Stiffness matrix of the system

$\mathbf{K}^{\mathbf{t o t}}$ : Stiffness matrix of the system, including the Jacobian matrix

C: Damping matrix of the system

$\mathbf{F}_{\mathbf{N L}}$ : Nonlinear force vector (including friction and contact)

$\mathbf{F}_{\text {ext }}$ : External force applied on the pad

$\tilde{\mathbf{M}}$ : Mass matrix of the system after Craig \& Bampton reduction 
$\tilde{\mathbf{K}}$ : Stiffness matrix of the system after Craig \& Bampton reduction

$\tilde{\mathbf{C}}$ : Damping matrix of the system after Craig \& Bampton reduction

M: Mass matrix of the system used for interface modes calculation

$\hat{\mathbf{K}}$ : Stiffness matrix of the system used for interface modes calculation

Ĉ: Damping matrix of the system used for interface modes calculation

$\boldsymbol{\Phi}^{p}$ : Matrix of the fixed modes (Craig \& Bampton reduction)

$\Psi^{p}$ : Matrix of the constraint modes (Craig \& Bampton reduction)

$N_{b}$ : Number of interface modes

$N_{p}$ : Number of modes for the pad

$N_{d}$ : Number of modes for the disc

$N_{c}:$ Number of contact points

T: Transfer matrix used for Craig \& Bampton reduction

$\mathbf{T}_{\mathbf{2}}$ : Transfer matrix used for $\hat{\mathbf{K}}$ and $\hat{\mathbf{M}}$ calculation

$\mathbf{T}_{R}$ : Transfer matrix used for the real interface modes reduction

$\mathbf{T}_{r}$ : Right transfer matrix used for the complex interface modes reduction

$\mathbf{T}_{l}$ : Left transfer matrix used for the complex interface modes reduction

$\mu$ : Friction coefficient

$k_{L}$ : linear stiffness at the friction interface $\left(N \cdot m^{-1}\right)$

$k_{N L}$ : nonlinear stiffness at the friction interface $\left(N \cdot m^{-3}\right)$

$\mathbf{X}_{R}^{n}: n^{\text {th }}$ real interface eigenvector

$\mathbf{X}_{B r}^{n}: n^{\text {th }}$ right complex interface eigenvector

$\mathbf{X}_{B l}^{n}: n^{\text {th }}$ left complex interface eigenvector

$F_{\text {friction,pad }}^{i}$ : Contact force on the pad at the friction interface for the $i^{\text {th }}$ contact node along the tangential direction

$F_{\text {friction,disc }}^{i}$ : Contact force on the disc at the friction interface for the $i^{\text {th }}$ contact node along the tangential direction

$F_{\text {contact,pad }}^{i}$ : Contact force on the pad at the friction interface for the $i^{\text {th }}$ contact node along the normal direction 
$F_{\text {contact,disc }}^{i}$ Contact force on the disc at the friction interface for the $i^{\text {th }}$ contact node along the normal direction

$v_{r}$ : Relative velocity between the pad and the disc

$\mathbf{u}_{i}^{p}$ : Internal degrees of freedom for the pad

$\mathbf{u}_{i}^{d}$ : Internal degrees of freedom for the disc

$\mathbf{u}_{j}^{p}$ : Interface degrees of freedom for the pad

$\mathbf{u}_{j}^{d}$ : Interface degrees of freedom for the disc

$\boldsymbol{\xi}^{p}$ : Generalized degrees of freedom for the pad, after Craig \& Bampton reduction

$\boldsymbol{\xi}^{d}$ : Generalized degrees of freedom for the disc, after Craig \& Bampton reduction

$\mathbf{X}_{\mathbf{0}}$ : Equilibrium point of the original nonlinear brake system

$\mathbf{J}\left(\mathbf{X}_{\mathbf{0}}\right)$ : Jacobian matrix

$\lambda_{k}$ : Complex eigenvalue

$a_{k}$ : Real part of the complex eigenvalue $\lambda_{k}$

$\omega_{k}$ : Imaginary part of the complex eigenvalue $\lambda_{k}$

$\overline{\mathbf{X}}$ : Small perturbation at the operating point $\mathbf{X}_{\mathbf{0}}$

$\varepsilon_{a, k}:$ Error criterion on the real parts

$\varepsilon_{\omega, k}:$ Error criterion on the frequencies

\section{Introduction}

It is well known that brake noise has been a challenging issue for the automobile industry for decades and that friction-induced squeal in automotive brakes is still an increasing source of customer complaints.

Even if all brake squeal mechanisms are not fully understood [1, 2], extensive research has been conducted into predicting and eliminating brake squeal since the 1960s $[3,4,5$, $6,7,8,9,10,11,12]$. It is commonly accepted that squeal noise is initiated by instabilities due to stick-slip, variable dynamic friction coefficient, sprag-slip or the friction coupling between the brake pad and disc and the appearance of mode coupling, leading to selfexcited vibrations $[1,2,8,9,13]$.

Nowadays, engineers classically perform a Complex Eigenvalues Analysis (CEA) to predict the onset of instability in the case of numerical studies focusing on a source of annoying noise emission from the mode coupling phenomenon. If the real part of the complex frequency is positive, then the brake system is called "unstable" as the original vibrations around a static equilibrium grow exponentially over time. Thereby, 
self-excited vibrations may be generated which induces squeal noise. Even if CEA may lead to underestimation or over-estimation of the unstable modes observed in squeal noise [14], it is classically admitted that CEA is a fast and efficient strategy in order to provide the occurrences of brake squeal for an industrial finite element model. As discussed by Ouyang et al. [11], the computational efficiency of the CEA approach is appreciated although more realistic results can be achieved via the transient analysis if the system model is sufficient. In this present paper, the numerical study is restricted to the complex eigenvalue analysis (CEA). For the interested readers on the aspects related to self-excited vibrations and squeal noise, we refer to the following papers [14, 15, 16, 17, 18, 19].

Due to the rapid development in computer aided engineering systems, finite element analysis are now classically used in the modeling of brake systems and the analysis of brake squeal. However, the main difficulty associated with the application of finite element analysis to brake squeal is the computational cost and time. So, one of the real challenge for the automobile industry and one of the major interest among researchers are to be able to develop general numerical techniques and guidelines to construct reduced finite element models or surrogate models of the finite element model [20, 21, 22] which can produce results comparable with the original finite element model while offering a massive reduction of computational times and data storage. One of the reasons of using such finite element models with many degrees of freedom lies in the fact that we need to represent the geometrical detail of complex structures. However, for representative finite element models of structural dynamics, engineers are only interested on the validity of the dynamic model in a restricted frequency range of interest. These restrictions on the expected predictions of the structural dynamic model allow the creation of reduced order models. So, the goal of the following work is to present a generalized double modal synthesis method that allows the creation of reduced finite element models for predicting the stability analysis of a damped nonlinear brake system subjected to friction-induced vibration. This numerical technique is developed in order to drastically reduce the size of the original finite element model of the brake system, particularly where the two subsystems are connected (i.e. reduction of the frictional interface between the pad and disc). It combines the motion of classical modal reduction (Craig \& Bampton synthesis) and a condensation at the frictional interface by computing a reduced complex mode basis. This new formulation may be seen as an extension of the previous work of Monteil et al. [23] in order to propose a reduction method in a generalized manner: the model reduction developed in the present work considers not only the possibility of constructing a reduced complex mode basis but also an application for a nonlinear system with a nonzero equilibrium point.

The paper is organized as follows: firstly, a description of the finite element model of the simplified brake system and the classical stability analysis for brake squeal prediction are discussed. Secondly, explanations are given of the classical common Craig \& Bampton reduction and a new generalized double modal synthesis method. Finally, we illustrate the effectiveness and robustness of the proposed generalized double modal synthesis technique through numerical simulations. 

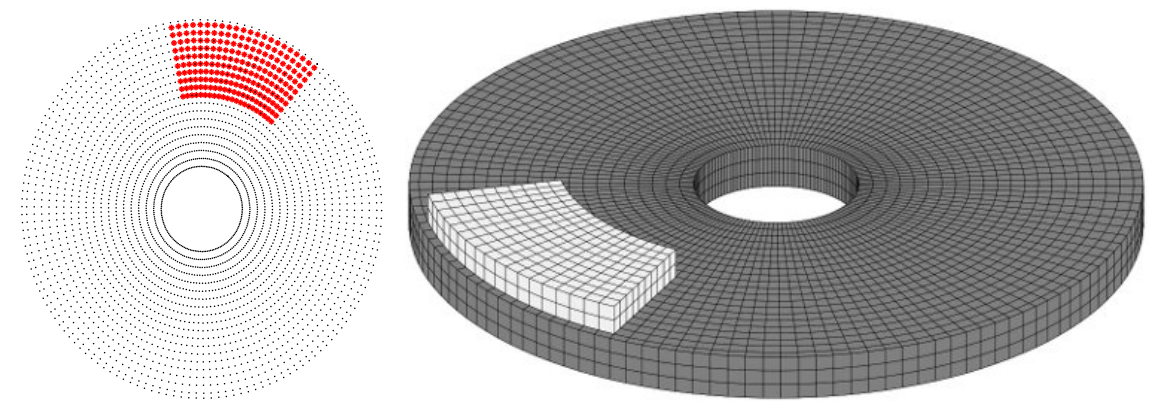

Figure 1: Finite element model and 220 contact points (red)

\begin{tabular}{lcc} 
& Disc & Pad \\
\hline Young modulus $E(\mathrm{~Pa})$ & $1.25 .10^{11}$ & $2.10^{9}$ \\
Density $\rho\left(\mathrm{kg} \cdot \mathrm{m}^{-3}\right)$ & 7200 & 2500 \\
Number of degrees of freedom $(\mathrm{dof})$ & 45000 & 2640 \\
Height $(\mathrm{m})$ & 0.019 & 0.0128 \\
$\mathrm{R}_{\text {int }}(\mathrm{m})$ & 0.034 & 0.091 \\
$\mathrm{R}_{\text {ext }}(\mathrm{m})$ & 0.151 & 0.147 \\
\hline
\end{tabular}

Table 1: Finite element details, material and geometric properties of the simplified brake system.

\section{Finite element model of the brake system and stability analysis}

In this section, we first present the finite element model of the brake system considered in the paper. Then, the hypothesis concerning the contact law and the damping are detailed. Finally, the classical stability analysis of an equilibrium point for the nonlinear brake system is briefly introduced.

\subsection{Finite element model}

Figure 1 shows the finite element model of the brake system under consideration. It represents a simplified brake consisting of a disc and a pad that are the two main components contributing to squeal. 220 contact nodes are distributed equally on the frictional interface between the pad and the disc surface, as shown in Figure 1. This choice of the 220 spaced contact points takes into account the fact that the interface refinement has a strong impact on the estimation of the stability analysis in terms of both the frequency and divergence rate. A convergence study versus the interface refinement (i.e. the number contact points at the frictional interface) has been conducted on this point (not presented in the paper). The hydraulic pressure is directly applied to the back-plate of the pad. The pad is in-plane fixed and the inner surface of the disc is clamped. Details on the finite element model, geometric and material properties are given in Table 1.

The set of equation describing the dynamic brake system can be written by:

$$
\mathbf{M X}+\mathbf{C X}+\underset{5}{\mathbf{K X}}=\mathbf{F}_{\mathrm{NL}}+\mathbf{F}_{\mathrm{ext}}
$$


where $\mathbf{X}$ is the generalized displacement vector while the dot denotes derivative with respect to time. $\mathbf{M}, \mathbf{C}$ and $\mathbf{K}$ are the mass, damping and stiffness matrices, respectively. In this study, modal damping is considered, and the damping coefficients $\xi_{k}$ are fixed at $0.1 \%$ for each mode. For the reader's comprehension, the role of damping and the structure of the damping matrix that are two extremely important issues in the squeal problem are not discussed in the present paper. For more information of the influence of damping, please see the following papers [24, 25, 26, 27]. $\mathbf{F}_{\text {ext }}$ corresponds to the vector force due to hydraulic brake pressure applied to the back-plate of the pad. $\mathbf{F}_{\mathrm{NL}}$ defines the linear and non-linear contact forces at the frictional interface. Considering previous studies [14, 28], the nonlinearities at the friction interfaces between the disc and the pad are both the cubic non-linear terms and the possible loss of contact between nodes on the disc surface and nodes on the pad surface. For the $i^{t h}$ contact node, the global nonlinear contact force vectors at the friction interface along the normal direction can be written as follows

$$
\begin{gathered}
F_{\text {contact,pad }}^{i}=k_{L} \delta x_{i}+k_{N L}\left(\delta x_{i}\right)^{3} \quad \text { if } \quad \delta x_{i}>0 \quad(0 \text { otherwise }) \\
F_{\text {contact,disc }}^{i}=-k_{L} \delta x_{i}-k_{N L}\left(\delta x_{i}\right)^{3} \quad \text { if } \quad \delta x_{i}>0 \quad(0 \text { otherwise })
\end{gathered}
$$

where $\delta x_{i}$ is the relative displacement between the pad and the disc for the $i^{\text {th }}$ contact node in the direction normal to the contact surface (i.e. $\delta x_{i}=x_{p a d, i}-x_{d i s c, i}$ where $x_{p a d, i}$ and $x_{d i s c, i}$ are the displacements of the disc and the pad for the $i^{\text {th }}$ contact node, respectively). $k_{L}$ and $k_{N L}$ are the linear and nonlinear stiffnesses at the friction interface between the disc and the pad. Values of these linear and nonlinear stiffnesses are fixed at $9.10^{5} \mathrm{~N} . \mathrm{m}^{-1}$ and $4.10^{9} \mathrm{~N} \cdot \mathrm{m}^{-3}$, respectively. The contact force vectors at the friction interface (for the $i^{\text {th }}$ contact node) along the tangential direction are defined by

$$
\begin{aligned}
& F_{\text {friction,pad }}^{i}=\mu F_{\text {contact,pad }}^{i} \cdot \operatorname{sign}\left(v_{r}\right) \\
& F_{\text {friction,disc }}^{i}=\mu F_{\text {contact,disc }}^{i} \cdot \operatorname{sign}\left(v_{r}\right)
\end{aligned}
$$

where $v_{r}$ is the relative velocity between the pad and the disc and $\mu$ defines the friction coefficient that is assumed to be constant. The classical Coulomb's law is applied without any stick-slip motion. Hence the global nonlinear force $\mathbf{F}_{\mathrm{NL}}$ :

$$
\mathbf{F}_{\mathrm{NL}}=\mathbf{F}_{\text {contact }}+\mathbf{F}_{\text {friction }}
$$

\subsection{Complex eigenmodes and stability analysis}

The stability analysis can be performed by considering the linearized system at the equilibrium point $\mathbf{X}_{0}$ of the original nonlinear brake system.

First, we estimate the steady-state operating point $\mathbf{X}_{0}$ for the full set of static nonlinear equations:

$$
\mathbf{K X}_{0}=\mathbf{F}_{\mathrm{NL}}+\mathbf{F}_{\text {ext }}
$$

As the vector force $\mathbf{F}_{\mathrm{NL}}$ contains nonlinear terms, equation 7 has to be solved using a nonlinear solver (Matlab solver in this work). 
Then, stability is performed on the linearized equations for small perturbations at the operating point $\mathbf{X}=\mathbf{X}_{0}+\overline{\mathbf{X}}$, which leads to the following equation:

$$
\mathbf{M} \ddot{\mathbf{X}}+\mathbf{C} \dot{\overline{\mathbf{X}}}+\underbrace{\left(\mathbf{K}-\mathbf{J}\left(\mathbf{X}_{\mathbf{0}}\right)\right)}_{\mathbf{K}^{\text {tot }}} \overline{\mathbf{X}}=\mathbf{0}
$$

where $\mathbf{J}\left(\mathbf{X}_{\mathbf{0}}\right)$ is the Jacobian matrix that corresponds to the linearized expression of the non-linear contact and friction forces at the equilibrium point.

Finally, the complex eigenvalue analysis provides the stability of the equilibrium points. The eigenvalue problem corresponding to equation 8 can be written as follows:

$$
\left(\lambda_{k}^{2} \mathbf{M}+\lambda_{k} \mathbf{C}+\mathbf{K}^{\mathrm{tot}}\right) \Phi_{k}=\mathbf{0}
$$

where $\left(\lambda_{k}, \Phi_{k}\right)$ are the eigenvalues and eigenvectors of the problem. The eigenvalues $\lambda_{k}=a_{k}+i \omega_{k}$ are complex. The imaginary part $\omega_{k}$ defines the $k^{t h}$ pulsation, whereas the associated real part $a_{k}$ is related to the stability of the system. If the real part of all the eigenvalues of the system remains negative, the equilibrium point $\mathbf{X}_{\mathbf{0}}$ is stable. If at least one of the eigenvalues has a positive real part, instability is generated from the equilibrium point $\mathbf{X}_{\mathbf{0}}$.

\section{Modal reduction}

This section aims at presenting the reduction techniques used in this study. Two reduction steps will be proposed:

- the Craig \& Bampton technique to condense the two substructures (i.e. the disc and pad);

- the remaining physical degrees of freedom, corresponding to the disc and pad interface, are reduced using the generalized double modal synthesis method.

The proposed reduction procedure is illustrated in Appendix A.

\subsection{Craig \& Bampton reduction}

The Craig \& Bampton technique consists in building a reduced model using fixed interface modes and constraint modes. Convergence of Craig \& Bampton method for a similar finite element brake system has been previously discussed in [23].

The internal degrees of freedom $\left(\mathbf{u}_{i}^{p}, \mathbf{u}_{i}^{d}\right)$ of the substructures (pad denoted $p$ and disc denoted $d$ ) are expressed in function of generalized degrees of freedom $\left(\boldsymbol{\xi}^{p}, \boldsymbol{\xi}^{d}\right)$ and interface degrees of freedom $\left(\mathbf{u}_{j}^{p}, \mathbf{u}_{j}^{d}\right)$ :

$$
\begin{aligned}
& \mathbf{u}_{i}^{p}=\boldsymbol{\Phi}^{p} \boldsymbol{\xi}^{p}+\boldsymbol{\Psi}^{p} \mathbf{u}_{j}^{p} \\
& \mathbf{u}_{i}^{d}=\boldsymbol{\Phi}^{d} \boldsymbol{\xi}^{d}+\boldsymbol{\Psi}^{d} \mathbf{u}_{j}^{d}
\end{aligned}
$$


where $\boldsymbol{\Phi}^{p}$ and $\boldsymbol{\Phi}^{d}$ are the matrices of the first fixed interface modes of the pad and the disc, whereas $\boldsymbol{\Psi}^{p}$ and $\boldsymbol{\Psi}^{d}$ are the matrices of the constraint modes. This allows to define the following transfer matrix $\mathbf{T}$ :

$$
\left\{\begin{array}{c}
\mathbf{u}_{i}^{p} \\
\mathbf{u}_{j}^{p} \\
\mathbf{u}_{i}^{d} \\
\mathbf{u}_{j}^{d}
\end{array}\right\}=\underbrace{\left[\begin{array}{cccc}
\boldsymbol{\Phi}^{p} & \mathbf{0} & \boldsymbol{\Psi}^{p} & \mathbf{0} \\
\mathbf{0} & \mathbf{0} & \mathbf{I} & \mathbf{0} \\
\mathbf{0} & \boldsymbol{\Phi}^{d} & \mathbf{0} & \mathbf{\Psi}^{d} \\
\mathbf{0} & \mathbf{0} & \mathbf{0} & \mathbf{I}
\end{array}\right]}_{\mathbf{T}}\left\{\begin{array}{c}
\boldsymbol{\xi}^{p} \\
\boldsymbol{\xi}^{d} \\
\mathbf{u}_{j}^{p} \\
\mathbf{u}_{j}^{d}
\end{array}\right\}
$$

Hence the following reduced mass and stiffness matrices:

$$
\tilde{\mathbf{M}}=\mathbf{T}^{T} \mathbf{M T} \quad \text { and } \quad \tilde{\mathbf{K}}=\mathbf{T}^{\mathbf{T}} \mathbf{K}^{\mathrm{tot}} \mathbf{T}
$$

The remaining physical degrees of freedom are located at the boundary between the disc and the pad (i.e. the physical frictional interface between the disc and the pad). The aim of the next section is to condense these remaining physical degrees of freedom.

\subsection{Interface reduction based on complex eigenmodes}

The interface reduction considered is based on interface modes [29]. This reduction strategy was previously applied to a simplified brake system considering interface modes computed with $\mu=0$, which leads to real eigenmodes. In the present work, all the interface modes are computed for $\mu \neq 0$. This leads to consider complex modes in the stability analysis: right and left eigenmodes have to be computed in order to get the reduced system.

To compute the basis of eigenvectors of the double modal synthesis method, mass and stiffness matrices have to be condensed on the boundary degrees of freedom (i.e. the $\mathrm{pad} /$ disc frictional interface in our case). Let us introduce the following transfer matrix $\mathbf{T}_{2}$ :

$$
\left\{\begin{array}{c}
\boldsymbol{\xi}^{p} \\
\boldsymbol{\xi}^{d} \\
\mathbf{u}_{j}^{p} \\
\mathbf{u}_{j}^{d}
\end{array}\right\}=\underbrace{\left[\begin{array}{ll}
\mathbf{0} & \mathbf{0} \\
\mathbf{0} & \mathbf{0} \\
\mathbf{I} & \mathbf{0} \\
\mathbf{0} & \mathbf{I}
\end{array}\right]}_{\mathbf{T}_{2}}\left\{\begin{array}{l}
\mathbf{u}_{j}^{p} \\
\mathbf{u}_{j}^{d}
\end{array}\right\}
$$

The transfer matrix $\mathbf{T}_{2}$ allows to define the reduced matrices $\hat{\mathbf{M}}=\mathbf{T}_{2}^{T} \tilde{\mathbf{M}} \mathbf{T}_{2}$ and $\hat{\mathbf{K}}=\mathbf{T}_{2}^{T} \tilde{\mathbf{K}} \tilde{\mathbf{T}}_{2}$

Right and left eigenvectors are computed, respectively, using the two following equations:

$$
\begin{gathered}
\left(-\omega_{i}^{2} \hat{\mathbf{M}}+\hat{\mathbf{K}}\right) \mathbf{X}_{B r}^{i}=\mathbf{0} \\
\left(-\omega_{i}^{2} \hat{\mathbf{M}}^{T}+\hat{\mathbf{K}}^{T}\right) \mathbf{X}_{B l}^{i}=\mathbf{0}
\end{gathered}
$$


Then, the reduced system can be obtained by introducing the matrices $\mathbf{T}_{r}$ and $\mathbf{T}_{l}$ as follows:

$$
\begin{aligned}
& \mathbf{T}_{r}=\left[\begin{array}{ccc}
\mathbf{I} & \mathbf{0} & \mathbf{0} \\
\mathbf{0} & \mathbf{I} & \mathbf{0} \\
\mathbf{0} & \mathbf{0} & \mathbf{X}_{B r}
\end{array}\right] \\
& \mathbf{T}_{l}=\left[\begin{array}{ccc}
\mathbf{I} & \mathbf{0} & \mathbf{0} \\
\mathbf{0} & \mathbf{I} & \mathbf{0} \\
\mathbf{0} & \mathbf{0} & \mathbf{X}_{B l}^{T}
\end{array}\right]
\end{aligned}
$$

where $\mathbf{X}_{B r}$ is the matrix of the first modes $\mathbf{X}_{B r}^{i}$ and $\mathbf{X}_{B l}$ is the matrix of the first modes $\mathbf{X}_{B l}^{i}$. The eigenmodes of the system are then computed as follows:

$$
\left(\lambda_{i}^{2}\left(\mathbf{T}_{l} \tilde{\mathbf{M}} \mathbf{T}_{r}\right)+\lambda_{i}\left(\mathbf{T}_{l} \tilde{\mathbf{C}} \mathbf{T}_{r}\right)+\left(\mathbf{T}_{l} \tilde{\mathbf{K}} \mathbf{T}_{r}\right)\right) \boldsymbol{\Phi}_{\mathbf{i}}=\mathbf{0}
$$

Finally, the Complex Eigenvalue Analysis (CEA) can be applied on the reduced finite element model to investigate the stability analysis of the brake system under study.

Algorithms in universal formal pseudo-code are provided for the Double Modal synthesis on the case of both real and complex modes in Appendix A. The global sequence for the stability analysis of the nonlinear brake system with the classical modal reduction and the two possible condensations at the frictional interface are also given in Appendix A.

\section{Results}

In this section, we present the results of the stability analysis of the brake system for $\mu \in[0,1]$. As it will be shown later, three unstable modes (around $2000 \mathrm{~Hz}, 3400 \mathrm{~Hz}$ and $4600 \mathrm{~Hz}$ ) are present in the frequency range of interest (i.e. $[0,5000] \mathrm{Hz}$ ).

To summarize, three synthesis techniques and calculations will be performed and compared :

- the "reference" without any interface condensation (i.e. by applying the classical Craig \& Bampton reduction and using the matrices $\tilde{\mathbf{K}}$ and $\tilde{\mathbf{M}}$, see Section 3.1);

- a modal reduction using "real" eigenvectors [23];

- a modal reduction using "complex" eigenvectors, as described in Section 3.2.

At this stage, it is also important to remember that the present work also offers original features and extensions on other aspects compared to the previous study proposed by Monteil et al. [23]. In this paper, the mathematical-modeling at the frictional interface is more complex by considering a nonlinear stiffness (see equations 2, 3, 4 and 5). This leads to take into account a non-zero equilibrium point (by solving equation 8) and the contribution of Jacobian matrix (i.e. the linearized expression of the nonlinear forces around an equilibrium point). 
Figures $2 \mathrm{a}$ and $2 \mathrm{~b}$ show evolutions of the real and imaginary parts in the complex plan for the first unstable mode (around $2000 \mathrm{~Hz}$ ) by using the complex or real eigenvectors, respectively. It is observed that the use of 12 complex eigen-modes is enough to provide an error of less than $2 \mathrm{~Hz}$ for both double modal synthesis techniques. The two double modal synthesis based on real or complex eigenvectors can be efficient in order to undertake the stability analysis of a nonlinear system with non-zero equilibrium point. If we now compare specifically the two proposed approaches, it seems, however, that the results obtained using real eigenvectors are not as good as the results obtained by using complex eigenvectors. This fact is especially clear for 20 interface modes (compare Figures 2a and $2 \mathrm{~b})$.

Then, Figures $3 \mathrm{a}$ and $3 \mathrm{~b}$ give evolutions of the real and imaginary parts in the complex plan for the second unstable mode (around $3400 \mathrm{~Hz}$ ). Here the two proposed modal reduction methods give good results and the difference between these two techniques is hardly visible. For the two modal reductions, increasing the number of interface modes drastically enhances the approximation quality for both the real parts and the frequency. Considering 20 complex eigen-modes seems to be enough to provide a good estimation of the second unstable mode.

Finally, Figures $4 \mathrm{a}$ and $4 \mathrm{~b}$ show evolutions of the real and imaginary parts in the complex plan for the third unstable mode (around $4600 \mathrm{~Hz}$ ). In the latter case, it clearly appears that the new formulation gives much better results than the previous proposed modal reduction [23]. Indeed, the use of 12 complex eigen-modes is enough to provide very good results if the modal reduction with complex eigenvectors is used (as illustrated in Figure 4a). On the contrary, the modal reduction based on real eigenvectors is not able to provide a suitable approximation of the unstable mode. More than 20 eigenvectors will be necessary in order to obtain a good prediction of the third unstable mode (this fact will be discuss in detail later in the paper). So the advantage of considering complex eigenmodes for double modal synthesis technique is clearly illustrated for this last case.

In conclusion, we have shown that calculations using real and complex eigenvectors can allow a good estimate of the unstable modes for a nonlinear system with non-zero equilibrium point. The effectiveness of the generalized double modal synthesis method with complex eigenmodes has been highlighted: this latter allows to obtain very convincing results for a small number of interfaces modes while the previous double modal synthesis method [23] does not work in all cases very well for the same number of interfaces modes.

For the interested readers, Figures 5 show the frequencies of the interfaces modes by increasing the number of interfaces modes from 5 to 50 . This data allows a better understanding of the system under study plus information on the frequency of interface modes compared to the frequency of the three unstable modes. For example, it may be noted that the first fifty interface modes have a lower frequency than the frequency of the second unstable mode. Moreover, Figures $5 \mathrm{~b}$ and $5 \mathrm{a}$ give evolutions of the frequencies for the three unstable modes (in the case of $\mu=1$ ) versus the number of interfaces modes for the double modal synthesis method with real and complex eigenmodes, respectively. 
First it can be seen that modal convergence occurs significantly more slowly in the case of the use of real eigenvectors than in the case of complex eigenvectors (i.e. the double modal synthesis method with real eigenmodes requires a higher number of interfaces modes). This is especially noticeable for the third unstable mode for which a decrease in the frequency is still visible: indeed, it appears that the results by using the double modal synthesis method with real eigenmodes have not yet converged completely even if 50 interfaces modes are kept. We can also see that a small jump is visible when the $20^{t h}$ interface modes is added. This vibration mode (which is around $1000 \mathrm{~Hz}$ ) provides an additional contribution in term of mode shape which allows an improvement in the prediction of the three unstable modes.

Now we propose to undertake more precisely a convergence study for each double modal synthesis method in order to compare more accurately these two approaches. This convergence study will be conducted for a number of interface modes ranging from 20 and 30. This choice is only guided by the fact that it was previously seen that an acceptable convergence is obtained with 20 interface modes and it allows to carefully compare the two proposed modal reduction techniques. In order to quantify the quality of the convergence, two criteria that are based on the relative error criterion between imaginary and real parts for the unstable modes in the frequency range of interest are proposed. Error on the real parts (i.e. divergence rate) of the $k^{t h}$ unstable mode is given by

$$
\varepsilon_{a, k}=\frac{a_{k}-\tilde{a}_{k}}{a_{k}}
$$

where $a_{k}$ is the real part of the $k^{t h}$ unstable mode for the "reference" (i.e. computed without any interface condensation) and $\tilde{a}_{k}$ the approximate real parts of the $k^{\text {th }}$ unstable mode calculated via the real interfaces modes or the complex interface modes. The calculation of error on the frequencies for the $k^{t h}$ unstable mode is defined by the following relation

$$
\varepsilon_{\omega, k}=\frac{\omega_{k}-\tilde{\omega}_{k}}{\omega_{k}}
$$

where $\omega_{k}$ is the "reference" " pulsation of the $k^{t h}$ unstable mode (i.e. pulsation of the system without any interface condensation) and $\tilde{\omega}_{k}$ is the approximate pulsation of the $k^{\text {th }}$ unstable mode calculated via the real interfaces modes or the complex interface modes.

Figure 6 and Tables 2 and 3 show the convergence of both double modal synthesis techniques for $\mu=0.5$ by increasing the number of interface modes from 20 to 30 for the three unstable modes. For more than 20 modes, both double modal synthesis techniques are efficient, but the advantage of the new proposed double modal synthesis technique is clearly visible: in all cases, errors on the real and imaginary parts made with the new reduction method are below those calculated by using the previous double modal synthesis method. Results follow the same trend for $\mu=1$, as illustrated in Figure 7 and Tables 4 and 5, thus demonstrating the efficacy of using the new proposed modal reduction with complex eigenvectors. It can be noted that the difference between the two double modal synthesis techniques is even greater. If we use complex interface 
modes (with a number of eigen-modes between 20 and 30), almost no error is observed for the third unstable modes, while if we use real interface modes more than $1 \%$ is obtained. This illustrates the fact that the modal double modal synthesis with complex modes takes better account of the friction contributions (i.e. the Jacobian matrix of the linearized expression of the non-linear contact and friction forces at the equilibrium point) so providing a better prediction of the squeal occurrence.

\begin{tabular}{lccc} 
Case & mode 1 & mode 2 & mode 3 \\
\hline 20 real eigenmodes & $1.8 \%$ & $2.6 \%$ & $1 \%$ \\
20 complex eigenmodes & $0.4 \%$ & $0.05 \%$ & $0.6 \%$ \\
\hline 30 real eigenmodes & $1.4 \%$ & $2 \%$ & $0.58 \%$ \\
30 complex eigenmodes & $0.4 \%$ & $0.2 \%$ & $0.6 \%$ \\
\hline
\end{tabular}

Table 2: Real vs complex modes: error $\varepsilon_{a, k}$ for $\mu=0.5$ (cf. equation 19) - three first unstable modes

\begin{tabular}{lccc} 
Case & mode 1 & mode 2 & mode 3 \\
\hline 20 real eigenmodes & $0.026 \%$ & $0.05 \%$ & $0.35 \%$ \\
20 complex eigenmodes & $0.018 \%$ & $0.028 \%$ & $0.04 \%$ \\
\hline 30 real eigenmodes & $0.023 \%$ & $0.042 \%$ & $0.25 \%$ \\
30 complex eigenmodes & $0.018 \%$ & $0.028 \%$ & $0.04 \%$ \\
\hline
\end{tabular}

Table 3: Real vs complex modes: error $\varepsilon_{\omega, k}$ for $\mu=0.5$ (cf. equation 20) - three first unstable modes

\begin{tabular}{lccc} 
Case & mode 1 & mode 2 & mode 3 \\
\hline 20 real eigenmodes & $4.48 \%$ & $7.2 \%$ & $2 \%$ \\
20 complex eigenmodes & $0.15 \%$ & $0.35 \%$ & $0.2 \%$ \\
30 real eigenmodes & $3.2 \%$ & $5.1 \%$ & $0.46 \%$ \\
30 complex eigenmodes & $0.1 \%$ & $0.1 \%$ & $0.2 \%$ \\
\hline
\end{tabular}

Table 4: Real vs complex modes: error $\varepsilon_{a, k}$ for $\mu=1$ (cf. equation 19) - three first unstable modes

\begin{tabular}{lccc} 
Case & Error mode 1 & Error mode 2 & Error mode 3 \\
\hline 20 real eigenmodes & $0.041 \%$ & $0.057 \%$ & $1.21 \%$ \\
20 complex eigenmodes & $0.02 \%$ & $0.03 \%$ & $0.02 \%$ \\
\hline 30 real eigenmodes & $0.029 \%$ & $0.035 \%$ & $1 \%$ \\
30 complex eigenmodes & $0.02 \%$ & $0.028 \%$ & $0.02 \%$ \\
\hline
\end{tabular}

Table 5: Real vs complex modes: error $\varepsilon_{\omega, k}$ for $\mu=1$ (cf. equation 20) - three first unstable modes 


\section{Conclusion}

In this paper, a new interface reduction method that is based on the use of complex interface eigen-modes is proposed to predict the occurrence of squeal noise. Furthermore, the use of the double modal synthesis approach [23] was tested in the case of a nonlinear brake system with a non-zero equilibrium point.

All the numerical results indicated that the new proposed double modal synthesis technique gives a better prediction of the stability analysis of the brake system in comparison with the previous double modal synthesis approach (only based on real modes): a better convergence of the unstable modes in terms of real and imaginary parts is observed.

The calculation cost of the proposed method is nearly the same as the technique based on real eigen-modes. Nevertheless, the eigen-modes basis depends on $\mu$ : the basis has to be computed of each value of $\mu$, which can be quite costly if $\mu$ takes various values; in this case, the use of real eigen-modes can remain a good choice, even if the results are not as good as the new proposed double modal synthesis method.

\section{Appendix A. Reduction procedure}

This appendix provides the global sequence for the stability analysis of the nonlinear brake system with the classical modal reduction and the two possible condensations at the frictional interface. Algorithms in universal formal pseudo-code are provided for the Double Modal synthesis on the case of both real and complex modes. 


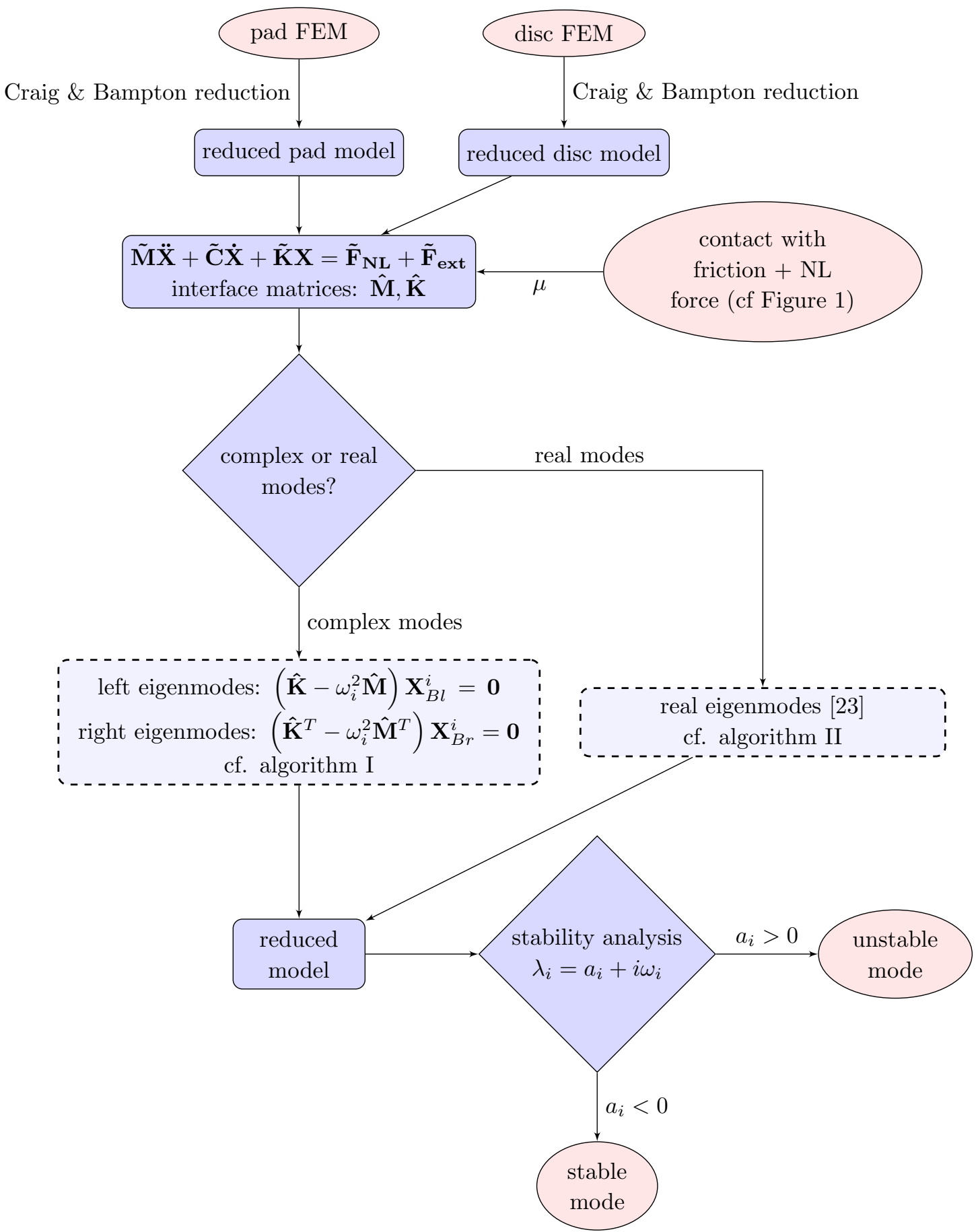




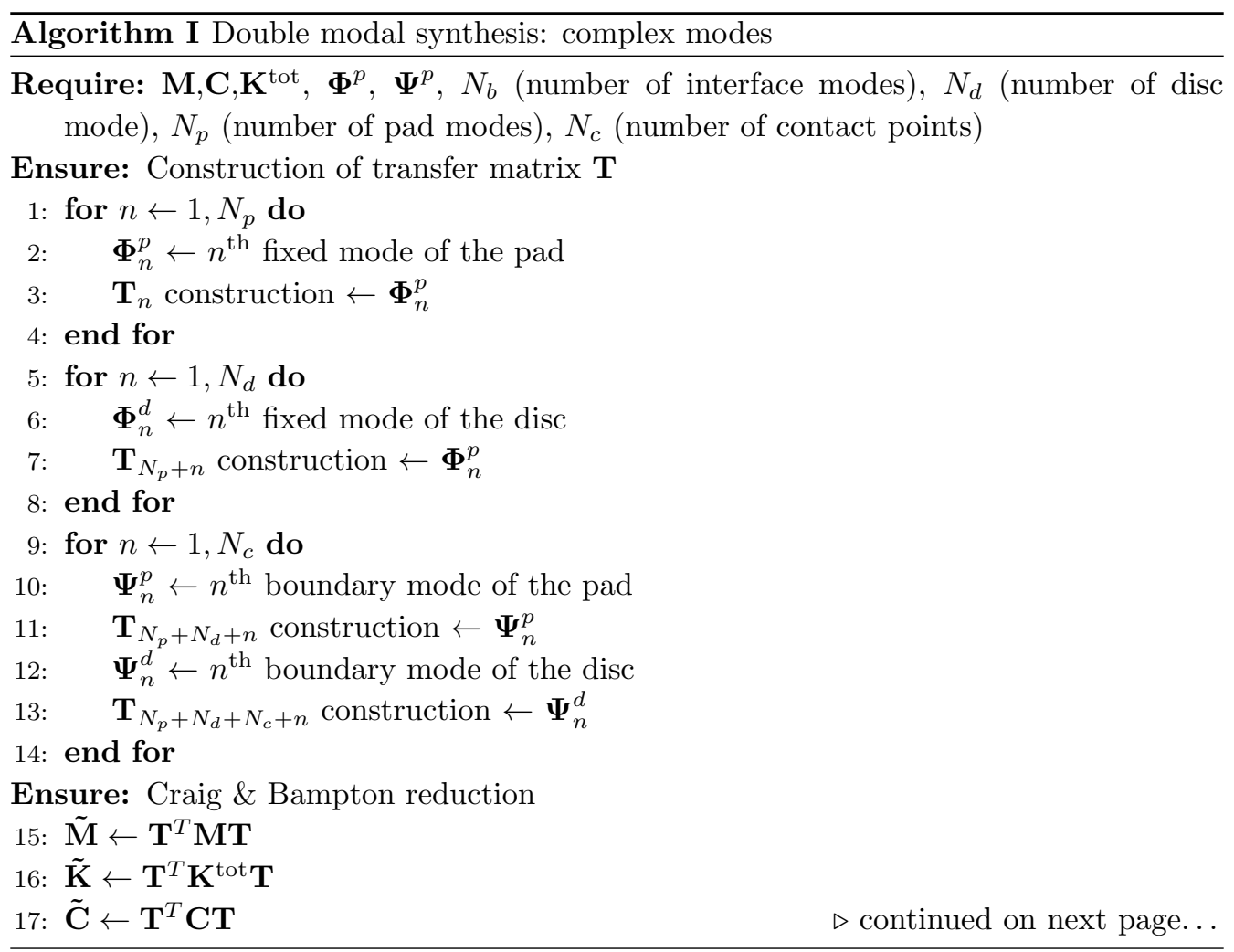




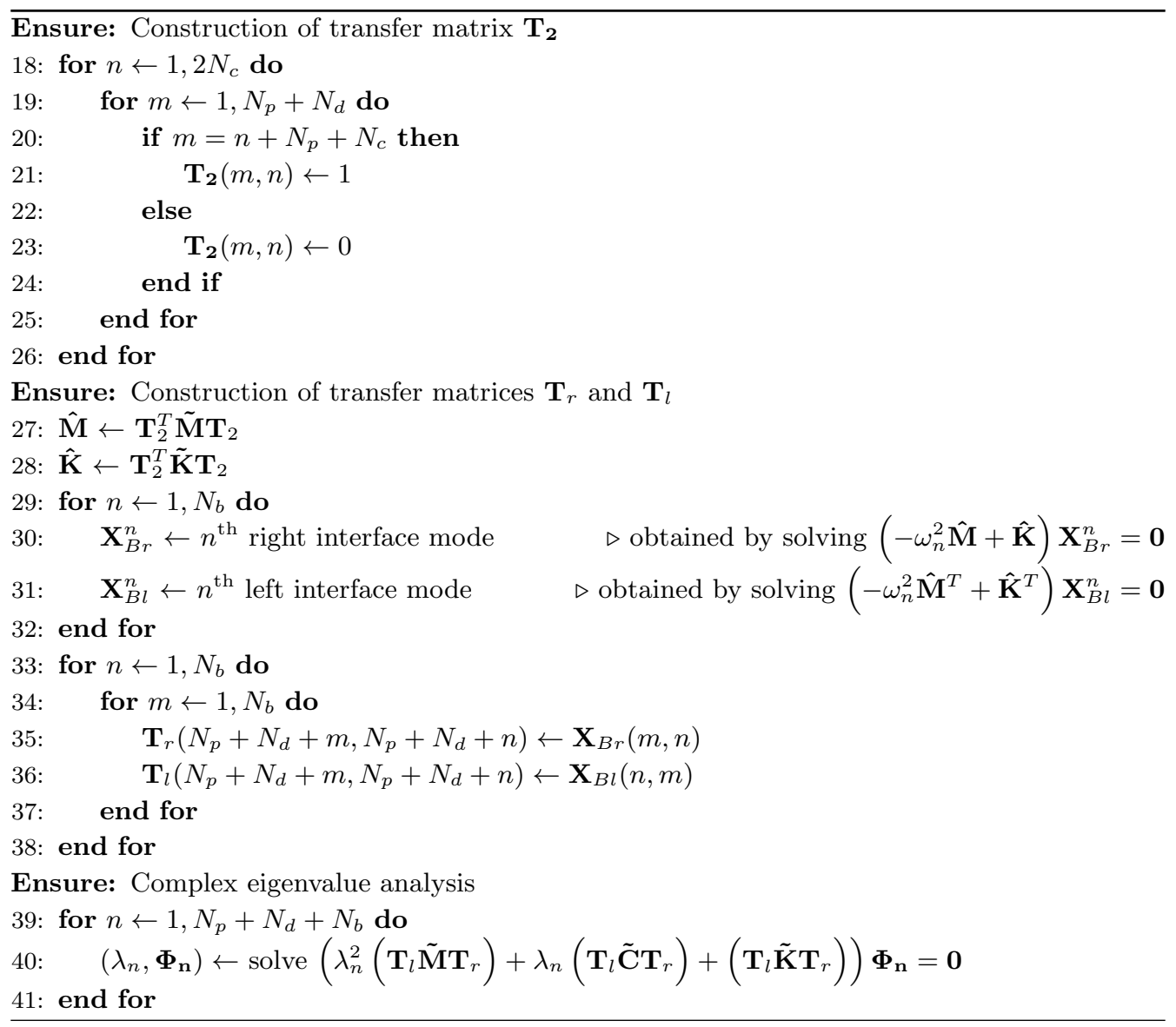




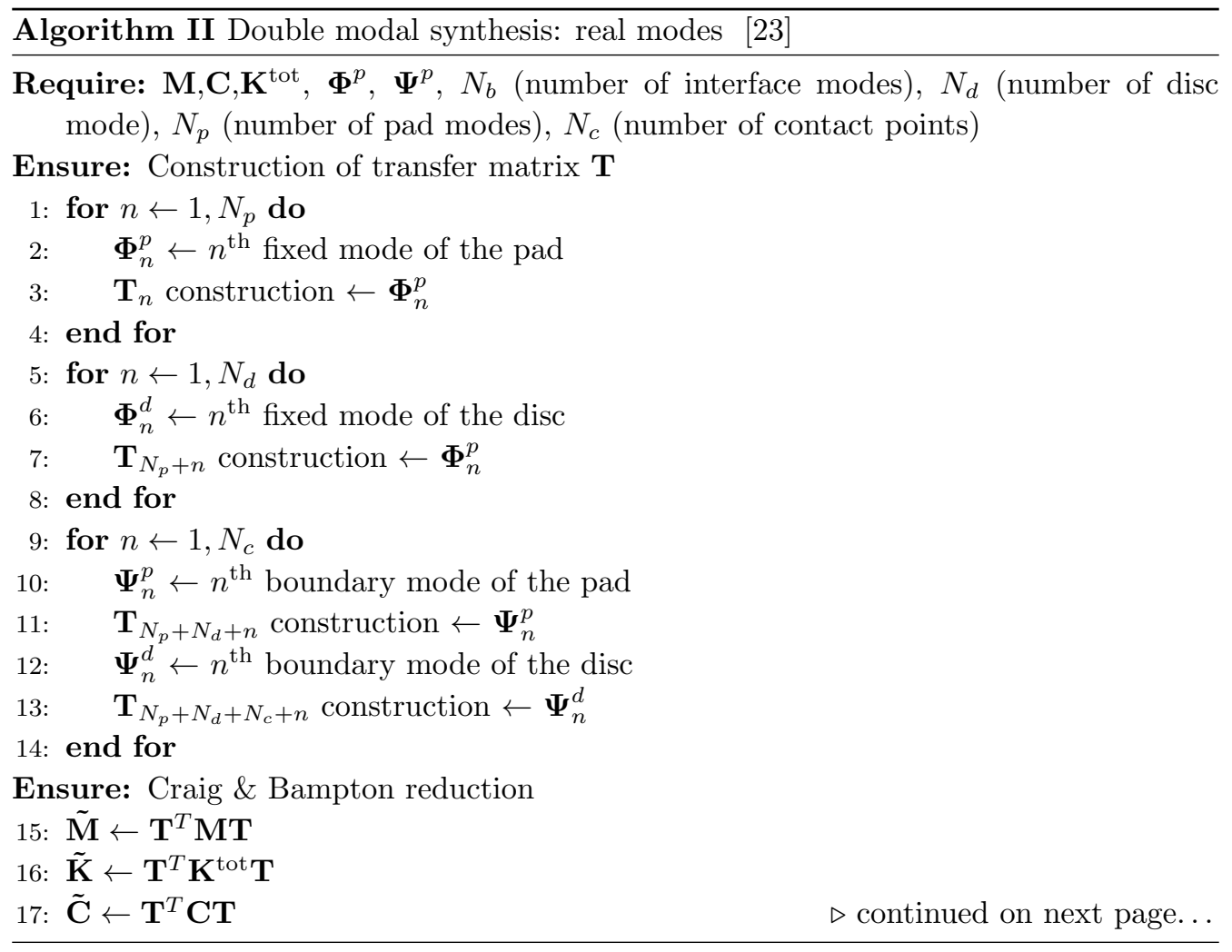




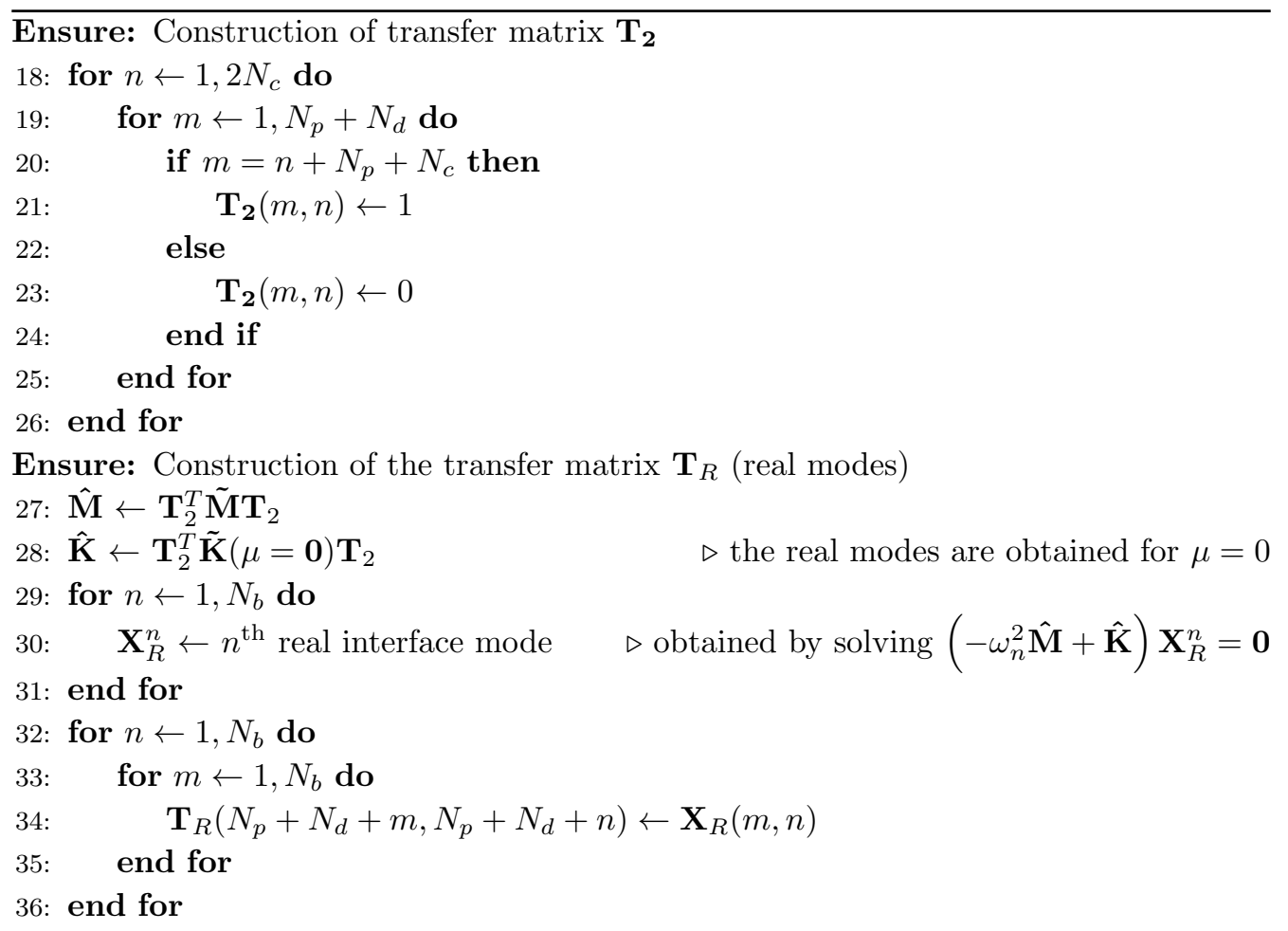

Ensure: Complex eigenvalue analysis

37: for $n \leftarrow 1, N_{p}+N_{d}+N_{b}$ do

38: $\quad\left(\lambda_{n}, \boldsymbol{\Phi}_{\mathbf{n}}\right) \leftarrow$ solve $\left(\lambda_{n}^{2}\left(\mathbf{T}_{R}^{T} \tilde{\mathbf{M}} \mathbf{T}_{R}\right)+\lambda_{n}\left(\mathbf{T}_{R}^{T} \tilde{\mathbf{C}} \mathbf{T}_{R}\right)+\left(\mathbf{T}_{R}^{T} \tilde{\mathbf{K}} \mathbf{T}_{R}\right)\right) \boldsymbol{\Phi}_{\mathbf{n}}=\mathbf{0}$

39: end for 

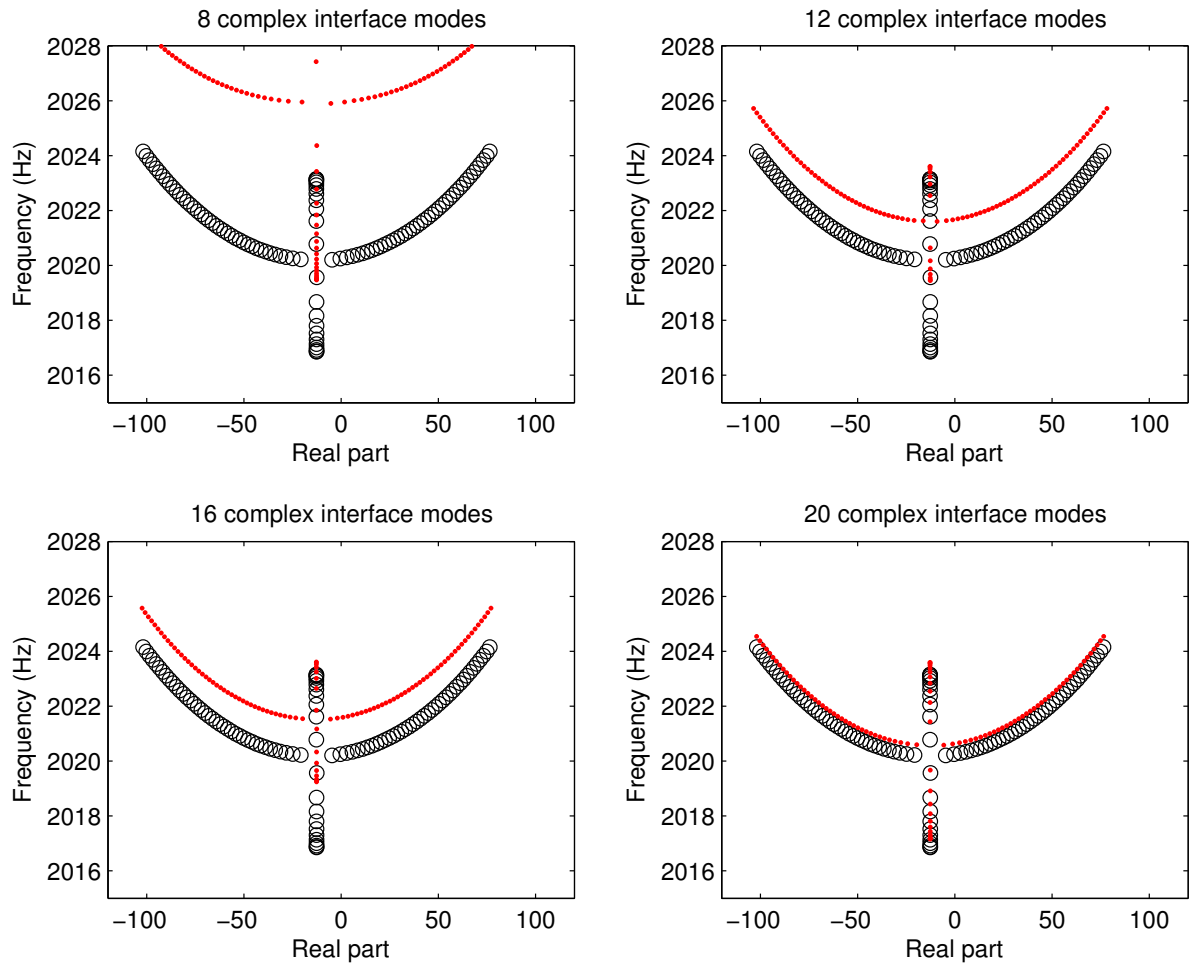

(a) complex interface modes.
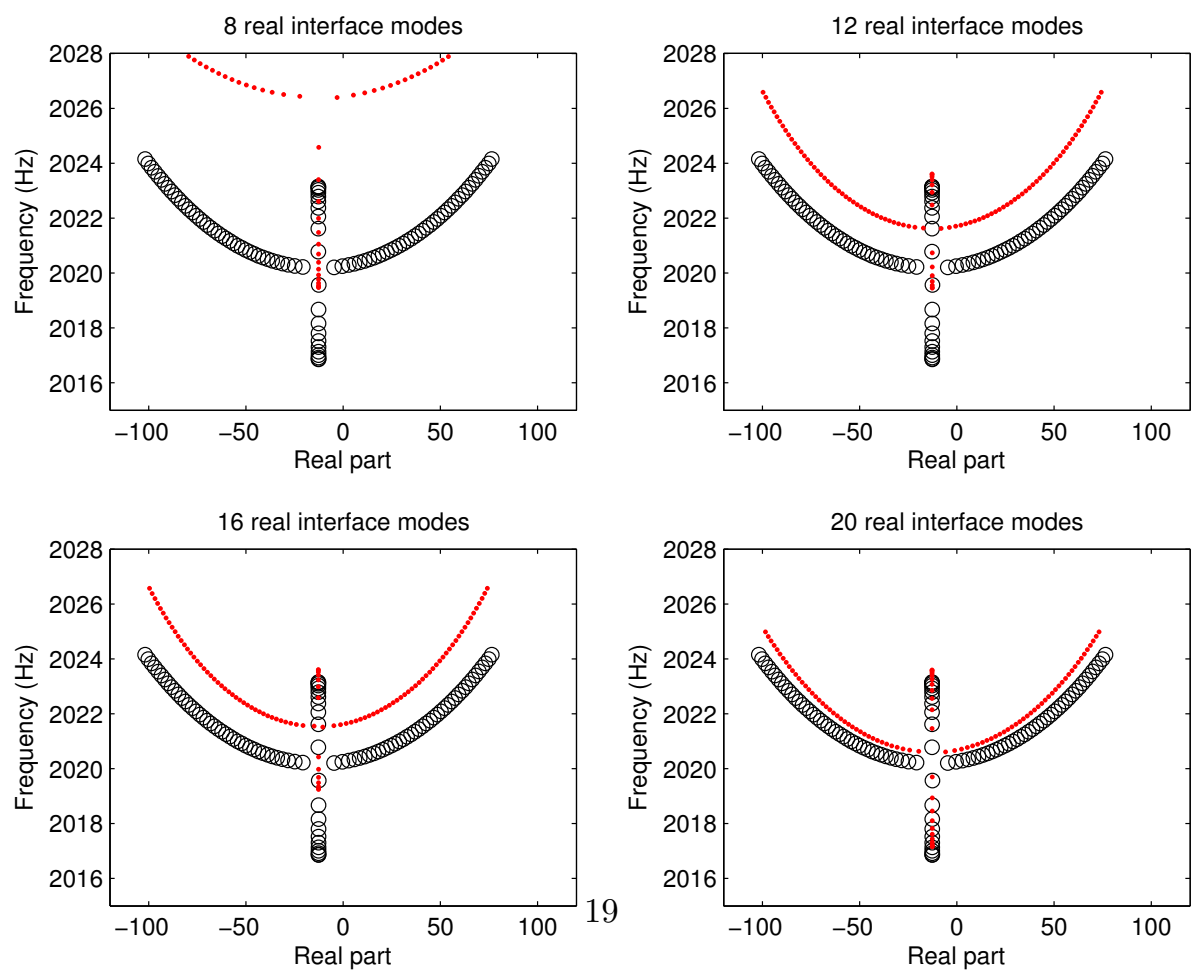

(b) real interface modes.

Figure 2: Evolution of the first unstable mode in the complex plan. o: reference, $\because$ interface modes. 

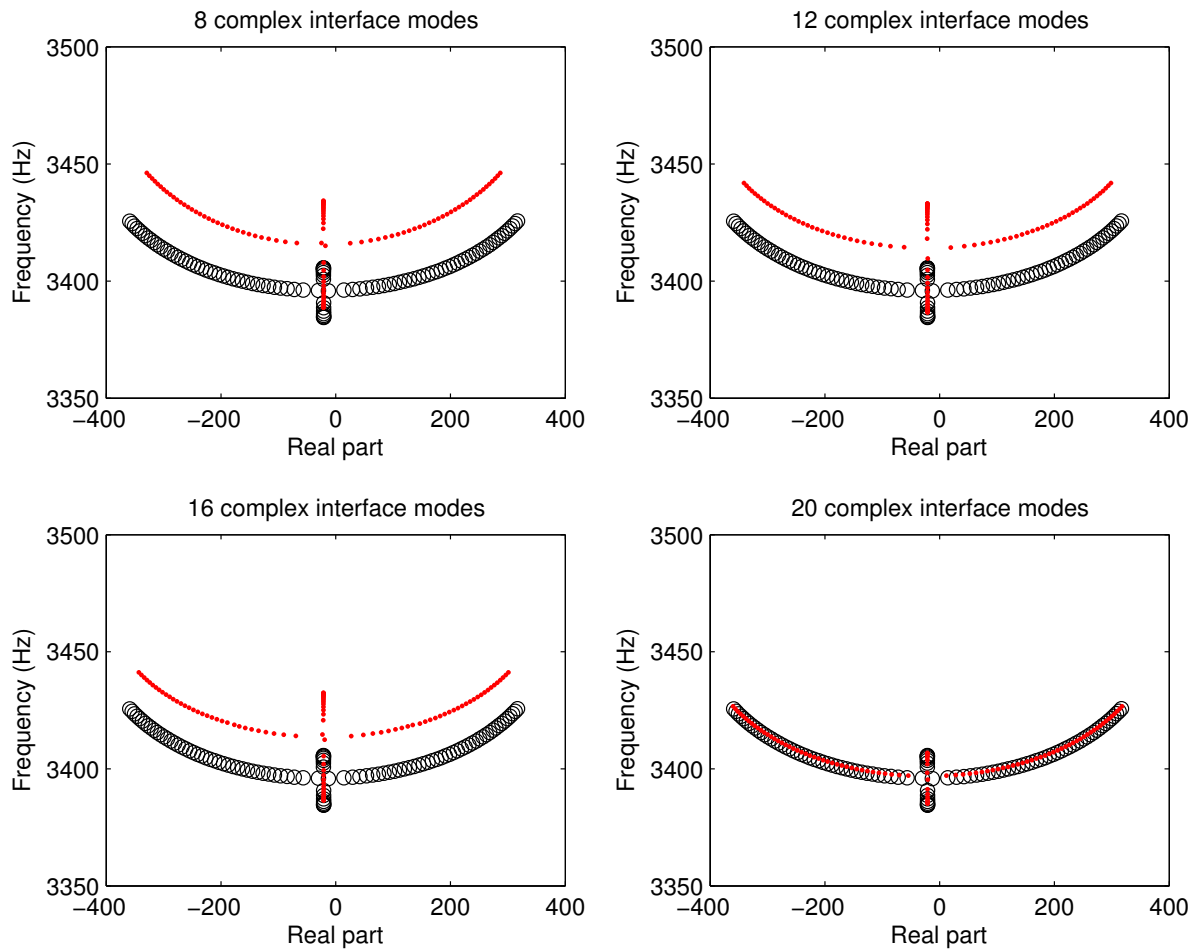

(a) Complex interface modes.
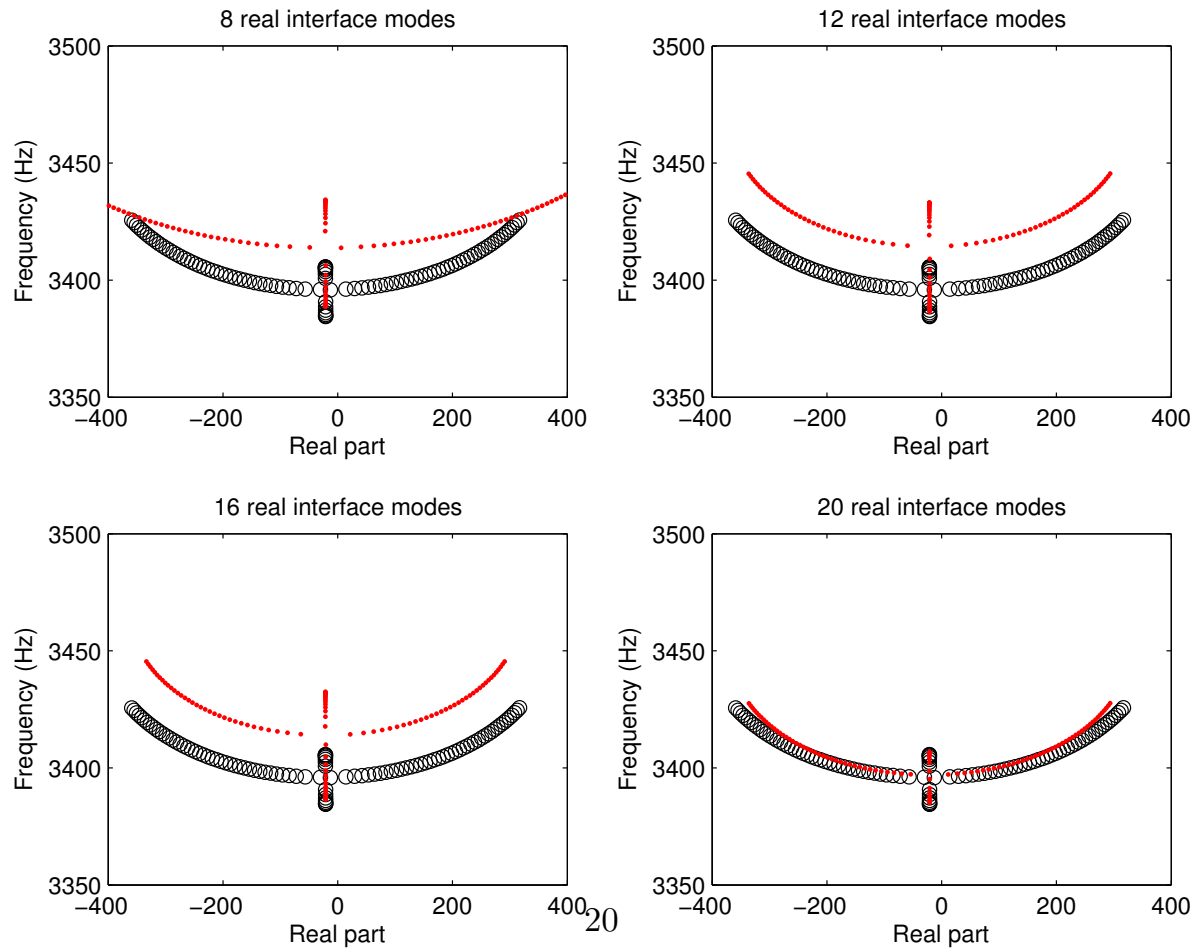

(b) Real interface modes.

Figure 3: Evolution of the second unstable mode in the complex plan. o: reference, $\because$ interface modes. 

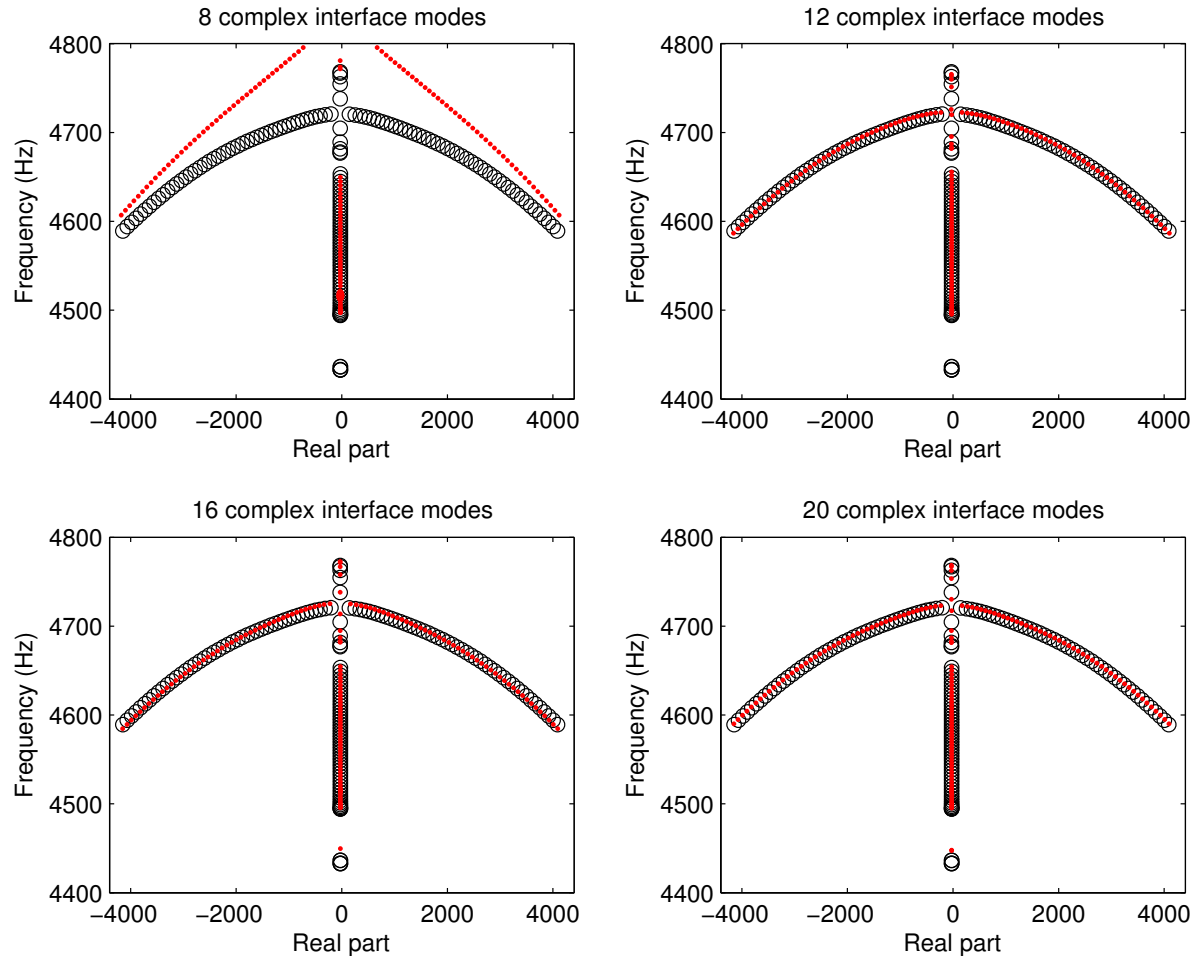

(a) Complex interface modes.
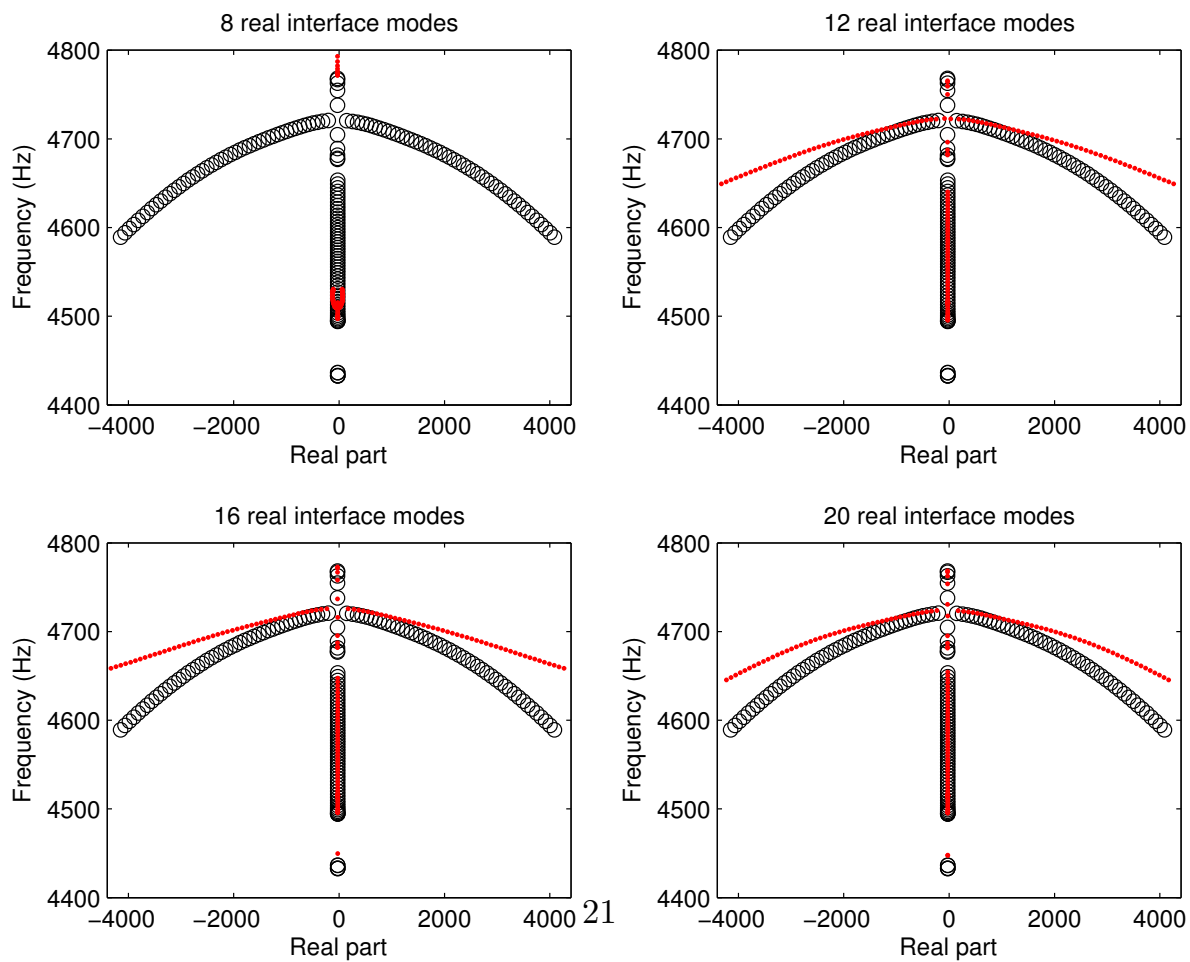

(b) Real interface modes.

Figure 4: Evolution of the third unstable mode in the complex plan. o: reference, $:$ interface modes. 


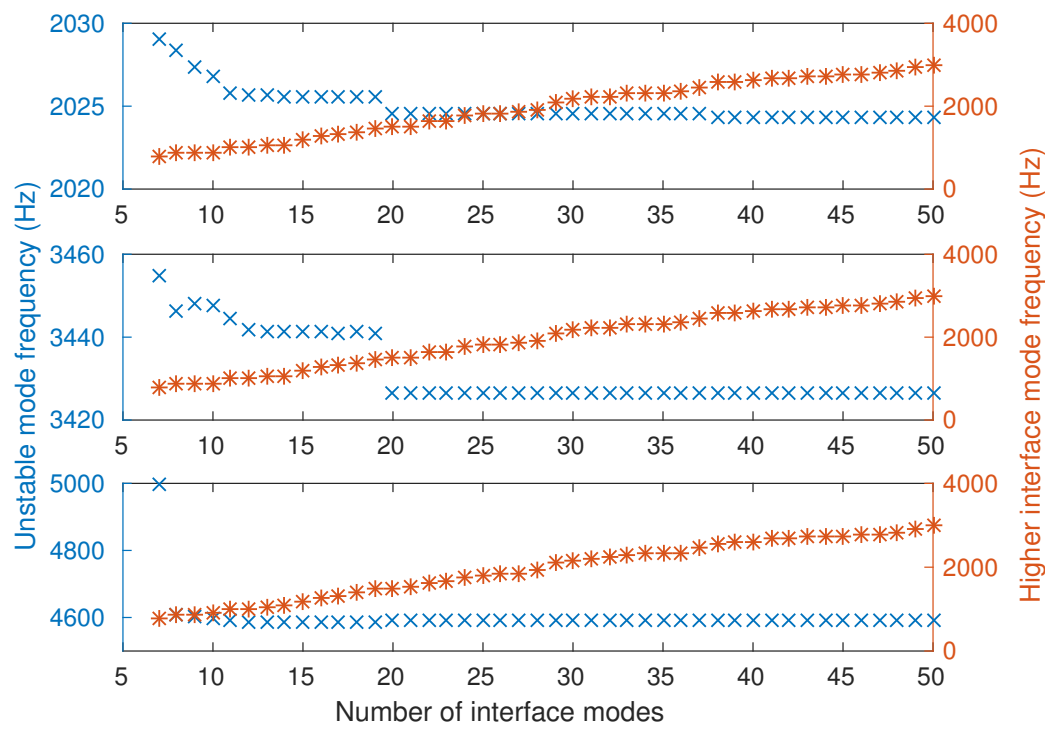

(a) Complex interface modes.

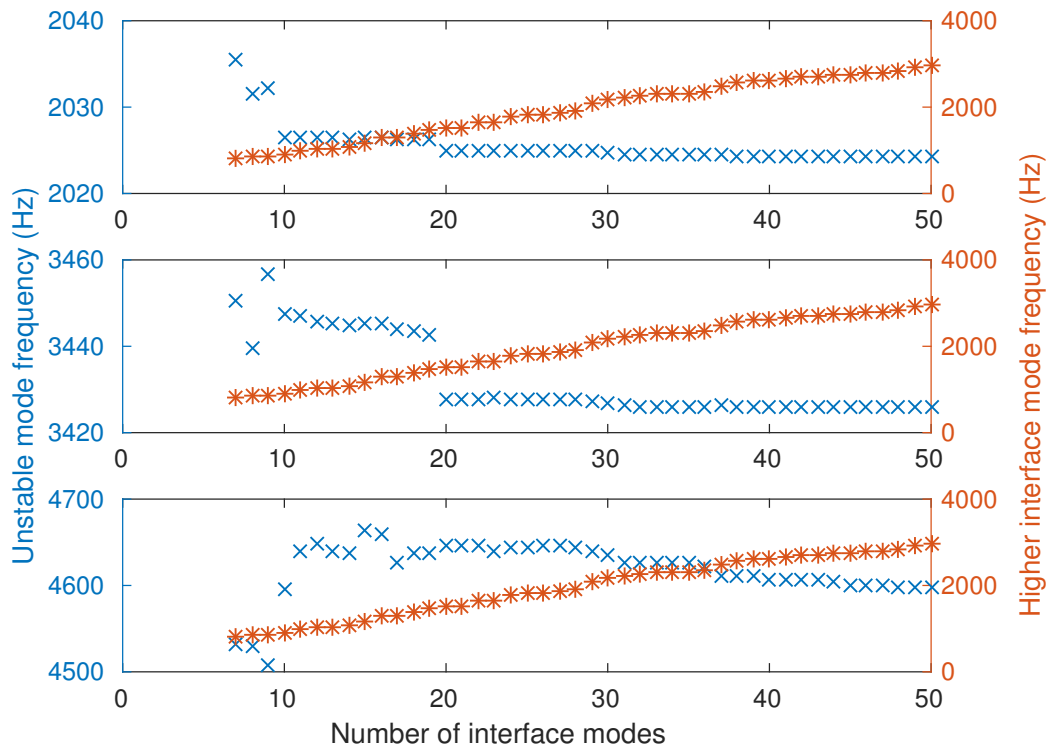

(b) Real interface modes.

Figure 5: Convergence study versus the number of interface modes and frequencies of the interfaces modes (from bottom to top : first, second and third unstable modes) 

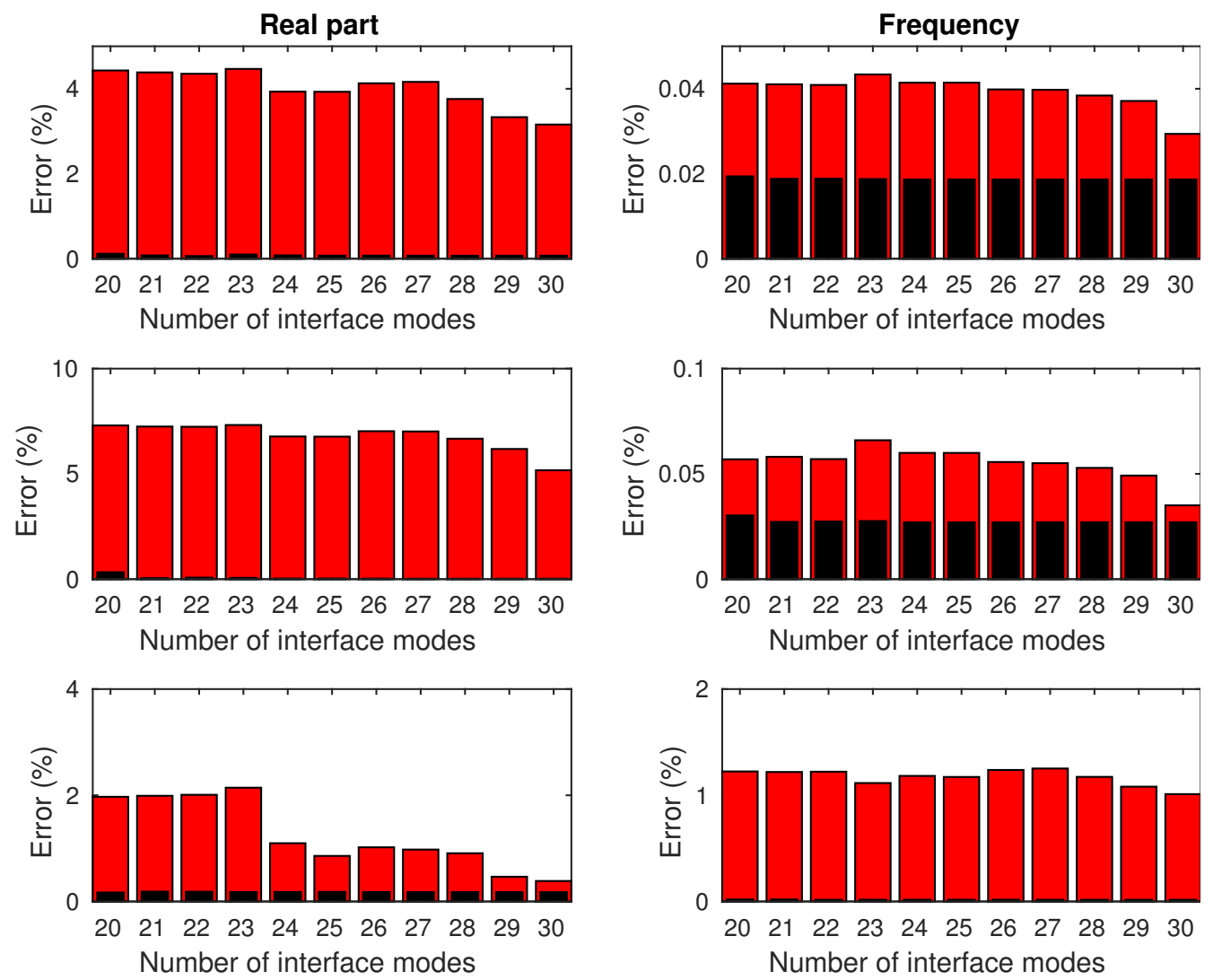

Figure 6: Convergence study for the real and imaginary parts of the three unstable modes (from bottom to top : first, second and third unstable modes for $\mu=0.5$ ) 

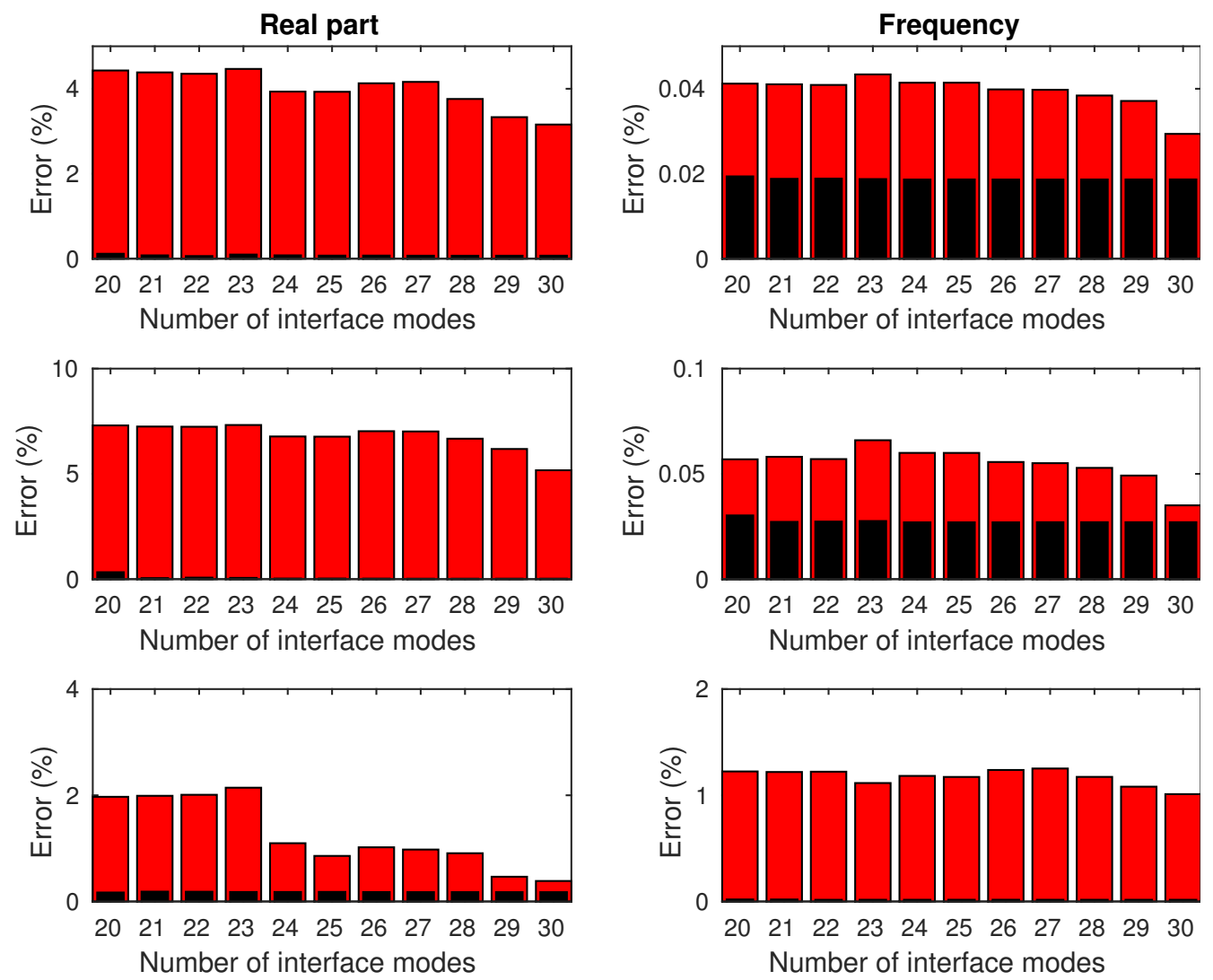

Figure 7: Convergence study for the real and imaginary parts of the three unstable modes (from bottom to top : first, second and third unstable modes for $\mu=1$ ) 


\section{Acknowledgement}

J.-J. Sinou acknowledges the support of the Institut Universitaire de France.

\section{References}

\section{References}

[1] R.A. Ibrahim. Friction-induced vibration, chatter, squeal and chaos part 1: mechanics of contact and friction. Applied Mechanics Review, 47:209-226, 1994.

[2] R.A. Ibrahim. Friction-induced vibration, chatter, squeal and chaos part ii : Dynamics and modelling. Applied Mechanics Review, 47:227-253, 1994.

[3] R.P. Jarvis and B. Mills. Vibrations induced by dry friction. Proceedings of the Institute of Mechanical Engineers, 178(32):847-866, 1963.

[4] S.W.E. Earles and C.K. Lee. Instabilites arising from the frictional interaction of a pin-disc system resulting in noise generation. Transaction ASME Journal of Engineering for Industry, 98(1):81-86, 1976.

[5] S.W.E. Earles and G.B. Soar. Squeal noise in disc brakes. Proceedings of the Institute of Mechanical Engineers Conference on Vibration and Noise in Motor Vehicles, Paper C100/71, 1971.

[6] N. Millner. An analysis of disc brake squeal. Society Automotive Engineer Technical Paper 780332, pages $1-11,1978$.

[7] M.R. North. A mechanism of disc brake squeal. 14th FISITA Congress, pages 1-9, 1972.

[8] R. Spurr. A theory of brake squeal. Proc. Auto. Div. Inst. Mech. Eng., 1:33-40, 1961.

[9] D.A. Crolla and A.M. Lang. Brake noise and vibration - state of art. Vehicule Tribology, Tribology Series, 18:165-174.

[10] N.M. Kindkaid, O.M. O'Reilly, and P. Papadopoulos. Automative disc brake squeal. Journal of Sound and Vibration, 267:105-166, 2003.

[11] H. Ouyang, W. Nack, Y. Yuan, and F. Chen. Numerical analysis of automative disc brake squeal : a review. International Journal of Vehicle Noise and Vibration, 1:207-231, 2005.

[12] A. Papinniemi, J.C.S. Lai, J. Zhao, and L. Loader. Brake squeal: a literature review. Applied Acoustics, 63(4):391-400, 2002.

[13] J.T. Oden and J.A.C. Martins. Models and computational methods for dynamic friction phenomena. Comput. Methods Appl. Mech. Eng., 52:527-634, 1985.

[14] J.-J. Sinou. Transient non-linear dynamic analysis of automotive disc brake squeal - on the need to consider both stability and non-linear analysis. Mechanics Research Communications, 37:96-105, 2010 .

[15] F. Massi, L. Baillet, O. Giannini, and A. Sestieri. Brake squeal: linear and nonlinear numerical approaches. Mechanical System Signal Processing, 21(6):2374-2393, 2007.

[16] F. Massi, Y.Berthier, and L. Baillet. Contact surface topography and system dynamics of brake squeal. Wear, 265(11-12):1784-1792, 2008.

[17] S. Oberst and J. C. S. Lai. Nonlinear transient and chaotic interactions in disc brake squeal. Journal of Sound and Vibration, 342:272-289, 2013.

[18] S. Oberst, J. C. S. Lai, and S. Marburg. Guidelines for numerical vibration and acoustic analysis of disc brake squeal using simple models of brake systems. Journal of Sound and Vibration, 332(9):2284-2299, 2013.

[19] K. Soobbarayen, S. Besset, and J-J. Sinou. Guidelines for numerical vibration and acoustic analysis of disc brake squeal using simple models of brake systems. Mechanical Systems and Signal Processing, 50:732-756, 2015.

[20] A. Nobari, H. Ouyang, and P. Bannister. Uncertainty quantification of squeal instability via surrogate modelling. Mechanical Systems and Signal Processing, 60-61:887-908, 2015.

[21] A. Nobari, H. Ouyang, and P. Bannister. Statistics of complex eigenvalues in friction-induced vibration. Journal of Sound and Vibration, 338:169-183, 2015. 
[22] Nechak L., F. Gillot, S. Besset, and J-J. Sinou. Sensitivity analysis and kriging based models for robust stability analysis of brake systems. Mechanics Research Communications, 69:13-145, 2015

[23] M. Monteil, S. Besset, and J.-J. Sinou. A double modal synthesis approach for brake squeal prediction. Mechanical Systems and Signal Processing, 70-71:1073-1084, March 2016.

[24] P. Hagedorn, M. Eckstei, E. Heffel, and A. Wagner. Self-excited vibrations and damping in circulatory systems. Journal of Applied Mechanics, 81:101009:1-9, 2014.

[25] O.N. Kirillov. Destabilization paradox due to breaking the hamiltonian and reversible symmetry. International Journal of Non-linear Mechanics, 42:71-87, 2007.

[26] O.N. Kirillov and A.P. Seyranian. Stabilization and destabilization of a circulatory system by small velocity-dependent forces. Journal of Sound and Vibration, 283:781-800, 2004.

[27] G. Fritz, J-J. Sinou, J-M. Duffal, and L. Jézéquel. Effects of damping on brake squeal coalescence patterns - application on a finite element model. Mechanics Research Communications, 34:181-190, 2007.

[28] J.-J. Sinou, N. Coudeyras, and S. Nacivet. Study of the nonlinear stationary dynamic of single and multi instabilities for disc brake squeal. International Journal of Vehicule Design, 51(1-2):207-222, 2008.

[29] S. Besset and L. Jezequel. Dynamic substructuring based on a double modal analysis. Journal of Vibration and Acoustics, 130(1), 2007. 